\title{
QUEEN'S
UNIVERSITY
BELFAST
}

\section{Whole wafer magnetostriction metrology for magnetic films and multilayers}

Hill, C. B., Hendren, W. R., Bowman, R. M., McGeehan, P. K., Gubbins, M. A., \& Venugopal, V. A. (2013).

Whole wafer magnetostriction metrology for magnetic films and multilayers. Measurement Science and

Technology, 24(4), [045601]. https://doi.org/10.1088/0957-0233/24/4/045601

\section{Published in:}

Measurement Science and Technology

\section{Document Version:}

Peer reviewed version

Queen's University Belfast - Research Portal:

Link to publication record in Queen's University Belfast Research Portal

Publisher rights

(C) 2013 IOP Publishing Ltd

\section{General rights}

Copyright for the publications made accessible via the Queen's University Belfast Research Portal is retained by the author(s) and / or other copyright owners and it is a condition of accessing these publications that users recognise and abide by the legal requirements associated with these rights.

Take down policy

The Research Portal is Queen's institutional repository that provides access to Queen's research output. Every effort has been made to ensure that content in the Research Portal does not infringe any person's rights, or applicable UK laws. If you discover content in the Research Portal that you believe breaches copyright or violates any law, please contact openaccess@qub.ac.uk. 


\title{
Whole wafer magnetostriction metrology for magnetic films and multilayers
}

\author{
C B Hill ${ }^{1, *}$, W R Hendren ${ }^{1}$, R M Bowman ${ }^{1, \#}$, P K McGeehin ${ }^{2}$, M A Gubbins ${ }^{2}$ and V A
} Venugopal $^{2}$

${ }^{1}$ Centre for Nanostructured Media, School of Mathematics and Physics, Queen's University Belfast, Belfast, BT7 1NN, UK

${ }^{2}$ Seagate Technology, Springtown, Northern Ireland, BT48 0BF, UK

Corresponding authors: * chill12@qub.ac.uk ${ }^{*}$ r.m.bowman@qub.ac.uk 


\begin{abstract}
The requirements for metrology of magnetostriction in complex multilayers and on whole wafers present challenges. An elegant technique based on radius of curvature deformation of whole wafers in a commercial metrology tool is described. The method is based on the Villari effect through application of strain to a film by introducing a radius of curvature. Strain can be applied tensilely and compressively depending on the material. The design, while implemented on 3" wafers, is scalable. The approach removes effects arising from any shape anisotropy that occurs with smaller samples, which can lead to a change in magnetic response. From the change in the magnetic anisotropy as a function of the radius, saturation magnetostriction $\lambda_{\mathrm{s}}$ can be determined.

Dependence on film composition and film thickness was studied to validate the radius of curvature approach with other techniques. $\lambda_{\mathrm{s}}$ decreases from positive values to negative values through an increase in $\mathrm{Ni}$ concentration around the permalloy composition, and $\lambda_{\mathrm{s}}$ also increases with a decrease in film thickness; in full agreement with previous reports. We extend the technique by demonstrating the technique applied to a multilayered structure. These results verify the validity of the method and are an important step to facilitate further work in understanding how manipulation of multilayered films can offer tailored magnetostriction.
\end{abstract}

Keywords: Magnetostriction, Magnetic Films, Whole Wafers, Multilayers 


\section{Introduction}

Magnetostriction of magnetic thin films is an area of interest in the magnetic recording industry. Very specific magnetic properties are required in elements such as recording heads; typically films with high saturation magnetization $\mathrm{B}_{\mathrm{s}}$, low coercivity and low magnetostriction. The need for low magnetostriction arises because when areal densities become greater, anisotropy effects become significant within magnetic recording heads. It is essential that they must be controlled in order to ensure that stresses are minimised in the presence of magnetic fields, and/or that magnetic properties will not be altered due to external stresses. Failure to control these two effects degrades the many operational performance indicators in a recording head [1-3].

At the same time, there has also been recent interest in magnetostriction control for micro-actuator applications, through the need to develop rare earth free alloy films that will give large magnetostriction. Recently, a re-visitation of $\mathrm{Co}_{1-\mathrm{x}} \mathrm{Fe}_{\mathrm{x}}$ has demonstrated that manipulation of parameters such as composition and temperature allow the magnetostriction to be manipulated to a significant degree [4].

Magnetostriction is either measured directly or indirectly. Direct measurements involve a dimensional change, whereas indirect measurements will measure a change in the magnetic property of the material as a result of strain. Magnetostriction then has to be calculated by relating it to the change in the anisotropy field. These indirect methods will yield $\lambda_{\mathrm{s}}$, the saturation magnetostriction, while direct methods will generally yield $\lambda_{\mathrm{e}}$, which is the field dependent magnetostrictive strain [5].

Direct methods involve application of a magnetic field which will bend the film-substrate bilayer due to magnetostrictive stress. Generally a uniform rotating field is applied to the sample, magnetizing it in one direction, while a magnetic field is applied in short pulses in the perpendicular direction; the directions depending on anisotropy being in or out of plane [6]. This produces a change in length of the sample where this deflection directly reflects the total film magnetization, and magnetostriction can be calculated from substrate deflection [7]. Up to a certain point, the $\lambda-\mathrm{H}$ response of these measurements will show a larger magnetostriction for a larger applied field value [8,9]. These deflections will also be time sensitive and so time and field are of great importance in direct measurements, as they will be dependent on these two factors.

Direct measurements, making use of the Joule effect, typically make use of mechanical or optical methods such as strain gauges [10], interferometers [11, 12] and the more sensitive capacitance technique [13-19]. Strain gauges and interferometers however, have the disadvantage where they require special sample preparation (e.g. ribbons, wires and thin film cantilevers) causing undue restrictions. It has been shown that the capacitance technique is able to measure samples of various lengths and even irregular shaped samples. For the shape of the beam used however, it is normally 
assumed that the width is small compared with the length so any curvature arising from displacement in the axis of the width can be neglected [17]. However in the case of a disc shaped sample, this assumption is not valid and curvature across the width of the sample cannot be ignored. The sample will not be under uniaxial stress and so capacitive measurements have limited applications for thin film wafers $[5,16-19]$.

Indirect measurement techniques, where as mentioned previously, a change in the magnetic response is measured, are viewed as more feasible and straightforward. There are three main techniques where strain can be applied to a sample; the three point bend, four point bend and applied radius of curvature. The three point bend technique involves upper and lower knife edges that exert a downward force on the sample. This technique has been employed in commercial instruments such as the SHB Instruments MESA inductive B-H looper whereby it can be equipped with an integrated magnetostriction three point assembly $[6,20]$. The sample is stressed by applying a downward force which is derived from a cantilever assembly at the back of the pickup, and can be varied with adjustable weights. This technique can be applied to whole wafers, however it has been noted that the use of knife edges in this manner do not strain the sample uniformly [21].

The four point bend consists of a sample with two load applicators and two supports. Upon bending, the two supports below the neutral axis of the sample create tension, and the two load applicators above this axis create compression [22]. The advantage of this method is that it offers a region of constant bending moment between the load applicators at the centre point, where in this region the stress would vary only along the direction of specimen thickness. Hence, for a magnetostriction measurement, if a pick up coil is around this region of the beam, the thickness-averaged B field measured here would be independent of the position and length of the coil [23]. This technique however, is based on the Euler-Bernoulli beam model and was developed for beam geometries; therefore could also be argued that it is not a suitable technique for whole wafers.

Alternatively, the radius of curvature technique will apply a uniform uniaxial strain to the whole sample. Unlike the three point bend where the strain can vary largely between the region above the knife edge and the two ends, the strain applied from the curved surface of a radius of curvature is uniform. This technique has been applied in conjunction with a vibrating sample magnetometer or the magneto-optic Kerr effect. A holder is generally made with a scheme to introduce a variable radius of curvature and a sample is fixed to this. Given the size of the stimulus/detection scheme (a laser spot in MOKE and pick-up coils in VSM) samples are usually diced into small strips. By straining a sample over a range of curvature radii allows the change in the anisotropy field to be obtained [21, 24-28].

In the indirect measurement of magnetostriction, the magneto optical Kerr effect is often used but the probing laser field will only penetrate a certain distance, approximately $24 \mathrm{~nm}[29,30]$ beyond the surface of the sample, thus it is only suitable for very thin films and not suitable for thicker or 
composite multilayered films. Further, the routine implementation is to apply a tensile strain exploiting the reflective nature of the films surface and frequently wafers are diced to fit the measurement tool. This can lead to an issue whereby dicing of a sample changes the anisotropy of a material and results in loss of the hard axis.

Taking all of the above into consideration, there is a need for a simple solution that addresses these three requirements - applicability to varying thickness of the thin film/multilayer, capability of tensile and compressive strain application, and the implementation of these conditions on whole wafers. In this paper we describe a universal extension to existing metrology that meets these requirements and allows the accurate measurement of magnetostriction of single films and multilayers in whole wafer samples.

\section{Experimental}

All samples were deposited in a load-locked UHV co-sputtering system with a base pressure of around $1 \times 10^{-9}$ mbar. In the process chamber both the $\mathrm{Ni}$ and $\mathrm{Fe}$ targets are positioned at an angle of $15^{\circ}$ to the substrate. $\mathrm{Ni}_{\mathrm{x}} \mathrm{Fe}_{100-\mathrm{x}}$ films were deposited onto $\mathrm{Si} / \mathrm{SiO}_{2}$ wafers at room temperature at a deposition rate of $3 \AA / \mathrm{s}$ and an Ar process gas pressure of $5 \times 10^{-3} \mathrm{mbar}$. The compositions of the films were controlled by altering the power of the magnetrons and by having a fixed deposition rate for each atomic composition percentage. The substrate was rotated at a speed of 20 RPM in order to obtain uniformity. Samples were also capped in a $5 \mathrm{~nm}$ layer of Ta to avoid oxidation. Samples were then ex-situ annealed for 2 hours in vacuum at $225^{\circ} \mathrm{C}$ with an applied field of $0.3 \mathrm{~T}$ and left to cool down over 12 hours.

The compositions and microstructures of films were revealed through EDX equipped for TEM and SEM. X-ray Diffraction was used to confirm crystalline orientation and lattice parameter calculation. Magnetic hysteresis loops were measured on a commercial SHB-instruments Model 110 BH-looper. The instrument was calibrated through cross reference with other magnetic metrology instruments in the laboratory already calibrated with NIST reference samples.

While recognising that SHB instruments provide a specialised three point bend magnetostriction pick up assembly for the MESA Inductive BH-looper [6], our model is not so equipped. Taking into account the limited dimensions within the instrument sample aperture, we have made an ingenious adaptation of the regular sample paddles that incorporates the radius of curvature to the wafer, with each paddle curvature ranging from $300 \mathrm{~mm}$ to $1500 \mathrm{~mm}$. This development allows us to ameliorate the challenges identified above, i.e. uniform sample stress for both tensile and compressive application, whole wafer capability obviating magnetic changes that can arise from sample dicing, and capability of handling layers of large thickness ranges as well as multilayer configurations. 
The paddles were precision machined from sheets of Tufnol using CNC milling. In this implementation the paddle was designed specifically for full 3" wafers (Figure 1). The wafer was positioned across the centre of the curvature and then mechanically secured by clamping it down at either end to ensure it was stressed fully across the radius. The distance to which the clamps are placed over the sample was kept consistent for each paddle, thus the strain was uniformly applied to the axis that is perpendicular to the applied magnetic field during annealing. The centre line of the paddle is co-incident with the pick-up assembly within the instrument.

Due to the simple nature of the paddle sample holder and the inductive detection scheme employed in the BH-Looper, the stress applied could be either tensile or compressive depending on how the sample was loaded onto the fixture. This is in marked contrast to MOKE based approaches. This is an important aspect as materials with different magnetostrictive signs will act differently depending on how the stress is applied [24].

Strain can be applied at any point during the fabrication process; however it is more beneficial to apply a strain during the measurement, as opposed to during deposition or annealing, as it allows a range of independent strains from a single film. Like most inverse measurement techniques, our method involves measurement of the change in the anisotropy field as a result of the applied strain. Further a result from one single film averaged over several strains will give more accuracy [31]. The change in anisotropy field $\mathrm{H}_{\mathrm{k}}$ as a result of applied strain was measured from the change in the hard axis loops between stressed samples of different radii of curvature. It is important that the hard axis is measured so that there is a linear response of the magnetization with the field outside of saturation regions [32]. We also note that the simplicity of the holder facilitates minor angular motion to identify the optimum orientation for closure of hard axis where achievable in samples. Through doing so this gives a more accurate representation of the anisotropy energy.

\section{Results and analysis}

To demonstrate the effectiveness of the design, initial measurements were focused on a range of Nickel-Iron samples with composition around the zero magnetostriction point, which from literature is taken to be approximately $\mathrm{Ni}_{80} \mathrm{Fe}_{20}$ [33]; films with higher Ni content have a negative magnetostrictive value, and films with lower Ni content will have a positive magnetostrictive value. Further, we highlight that accurate measurement of small values of magnetostriction around zero are most demanding [34].

The three compositions $\left(\mathrm{Ni}_{77} \mathrm{Fe}_{23}, \mathrm{Ni}_{80} \mathrm{Fe}_{20}\right.$ and $\left.\mathrm{Ni}_{83} \mathrm{Fe}_{17}\right)$ were prepared with expected magnetostrictions to be positive, close to zero, and negative, respectively. Figure 2 shows the effects of the application of strains to the films. By definition, for a positive magnetostrictive material the magnetization will be increased by tensile strain and for a negative magnetostrictive material the 
magnetization is decreased by tensile strain. Application of tensile stress to $\mathrm{Ni}_{77} \mathrm{Fe}_{23}$ creates an easier axis and application of compressive stress creates a harder axis, which is in agreement with how a positive material is defined. $\mathrm{Ni}_{83} \mathrm{Fe}_{17}$ demonstrates the reversal of this, as expected, while $\mathrm{Ni}_{80} \mathrm{Fe}_{20}$ shows little difference between the two.

A hard axis loop is required to calculate the magnetostriction and the magnetization must decrease when applying strain, so the sign of the magnetostrictive response of the material will determine whether compressive or tensile strain will be applied to obtain the anisotropy field. This is useful, particularly for films with unknown magnetostrictive values, as it will be clear on the first measurement whether tensile or compressive stress must be applied.

Radii of curvature used in this work ranged from 300 to $1500 \mathrm{~mm}$, where the smaller the radius the sample is bent over, the greater the applied stress. Three of these were chosen which demonstrated the largest change in anisotropy for our chosen material. This is shown clearly in Figure 3, where the field at which the magnetization reaches saturation is greater for a larger stress, which corresponds to a smaller radius. The strain applied remains within the elasticity limit of the material and so it is expected that the anisotropy field should depend linearly on applied stress.

The change in the anisotropy field between each of the stressed loops can be determined by fitting a polynomial of order of best fit to the normalised hysteresis loop [21]. Saturation magnetostriction can then be calculated by substituting the gradient value for each radius into the following equation:

$$
\lambda_{s}=\frac{d\left(H_{k}\right)}{d\left(\frac{1}{R}\right)} \frac{2 \mu_{0} M_{s}\left(1-v^{2}\right)}{3 t_{s} E_{s}}
$$

where $v$ is the Poisson's ratio of the substrate, $M_{s}$ is the saturation magnetisation, $t_{s}$ is the thickness of the substrate and $E_{s}$ is they Young's modulus of the substrate [21, 28].

The $\mathrm{Ni}_{\mathrm{x}} \mathrm{Fe}_{100-\mathrm{x}}$ films were investigated as a function of composition as above, and in addition, thickness. Figure 4 illustrates the variation of magnetostriction with respect to the Ni composition. The lowest Ni content of $77 \%$ has the highest positive magnetostriction value. As the Ni content increases the magnetostriction decreases in a linear trend, crossing over the zero value between approximately $80-81 \%$ and continues into the negative region. It is evident that the trend in our results and that found in other studies completely agree [33, 35-37].

Thicknesses of the three compositions from Figure 4 were also compared to understand how magnetostriction varies with film thickness. A positive, close to zero and negative composition were chosen and film thickness was varied from $10-60 \mathrm{~nm}$. Figure 5 shows this relation and there is a clear trend where magnetostriction decreases with an increase in thickness within this range, 
regardless of the magnetostrictive sign. This trend in results is in agreement with other published work $[15,27,38]$ confirming the effectiveness and efficacy of this new technique.

Finally, to clearly illustrate the suitability for more challenging samples that could not be measured by other indirect techniques such as MOKE, bilayered films of opposite magnetostricitve polarity were investigated. The films were deposited with the following structure A (x nm) / B (60-x nm), where A is $\mathrm{Ni}_{83} \mathrm{Fe}_{17}$ and $\mathrm{B}$ is $\mathrm{Ni}_{77} \mathrm{Fe}_{23}$. Figure 6 shows these results plotted against the thickness of layer $\mathrm{A}$, giving an expected trend where the magnetostriction changes from positive to negative as $\mathrm{x}_{\mathrm{A}}$ increases in thickness from 0 to $60 \mathrm{~nm}$. It appears from Figure 6 that at $\mathrm{x}_{\mathrm{A}}=30 \mathrm{~nm}, \lambda_{\mathrm{s}}$ reaches zero which would be the structure: A $(30 \mathrm{~nm}) / \mathrm{B}(30 \mathrm{~nm})$. This observed trend is somewhat similar to other published work [34] and is a good indication that multilayered systems can indeed be manipulated and measured with this technique.

\section{Conclusion}

The analysis concludes that the radius of curvature fixture to be used in conjunction with a BH-looper, and applied to full wafers, is a viable approach for magnetostriction measurement. Preliminary results agree with literature by illustrating that (a) there is a thickness dependence of NiFe magnetostriction, (b) there is a NiFe composition dependence, whereby depending on the magnetostrictive properties of the film, compressive or tensile stress must be applied, and (c) layering of positive and negative magnetostrictive materials can be altered to reach zero. The method presented has important advantages as it utilizes full wafers, is a simple, cost effective set up, and can be applied to both thicker films and multilayers. This sets a fundamental basis for further work, in particular with multilayered films, in an effort to prove that manipulation of these layers will allow control of magnetostriction.

\section{Acknowledgements}

We thank Seagate Technology (Ireland) for their financial support to establish ANSIN (www.ansin.eu). We thank Philip Orr for invaluable technical assistance. 

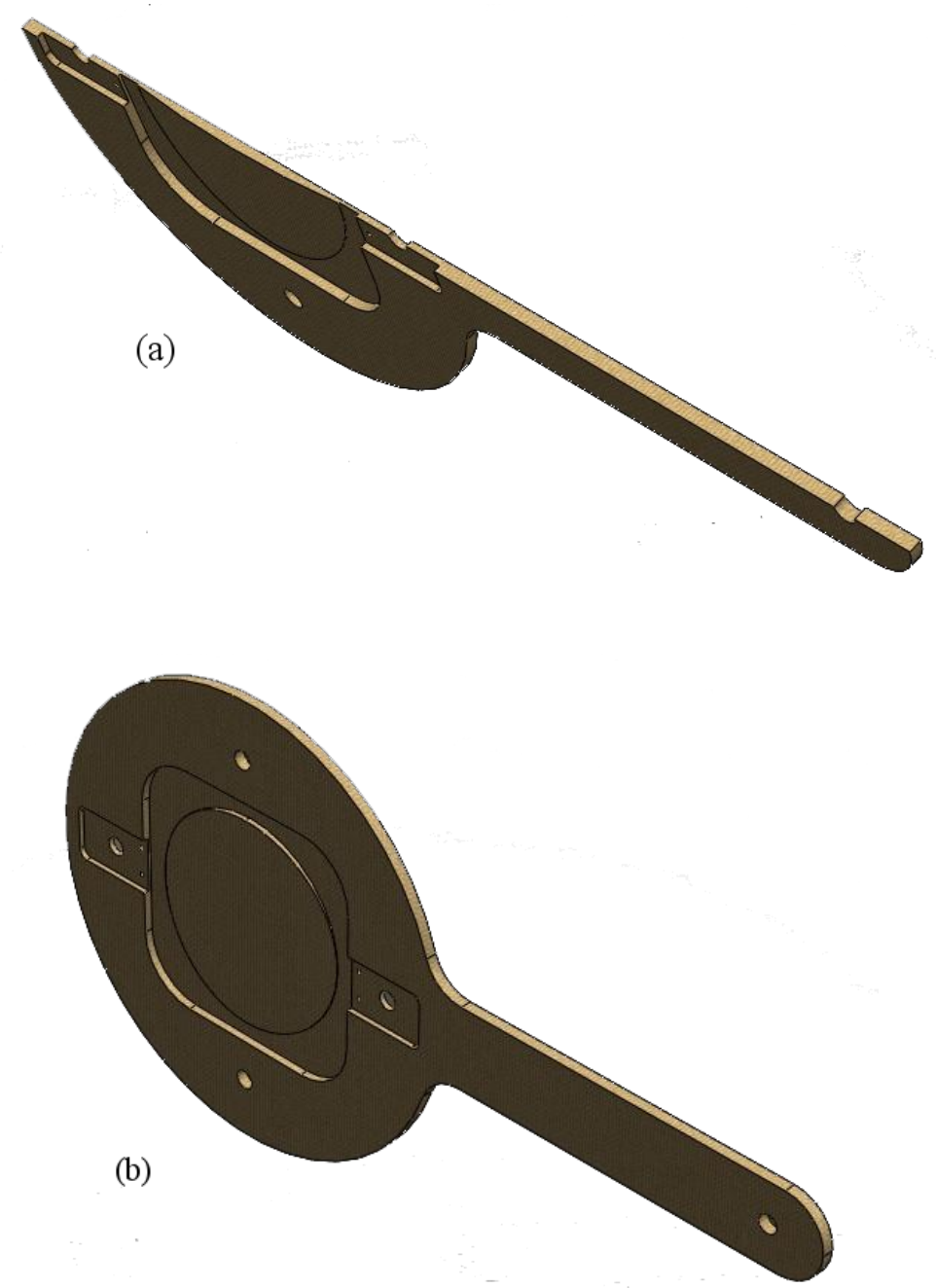

Figure 1:

Images of Tufnol paddle showing (a) cross section and (b) full view to be inserted in a BH-looper for a specific radius. 3" wafers are stressed and held in position with clamps where the stress may be tensile or compressive depending on how the sample is fixed. 

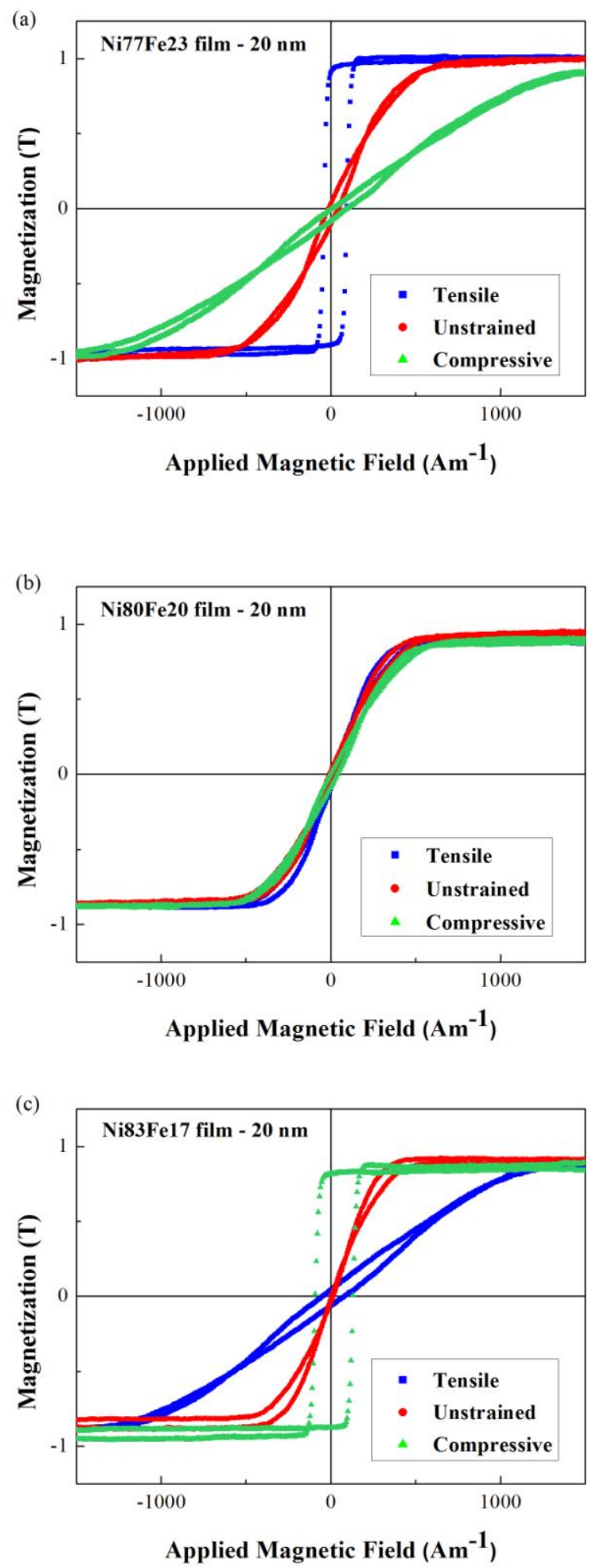

Figure 2:

Hysteresis loops in unstressed conditions and with equal applied tensile and compressive stress corresponding to a radius of curvature of $500 \mathrm{~mm}$ for (a) $\mathrm{Ni}_{77} \mathrm{Fe}_{23}$ (b) $\mathrm{Ni}_{80} \mathrm{Fe}_{20}$ (c) $\mathrm{Ni}_{83} \mathrm{Fe}_{17}$. 


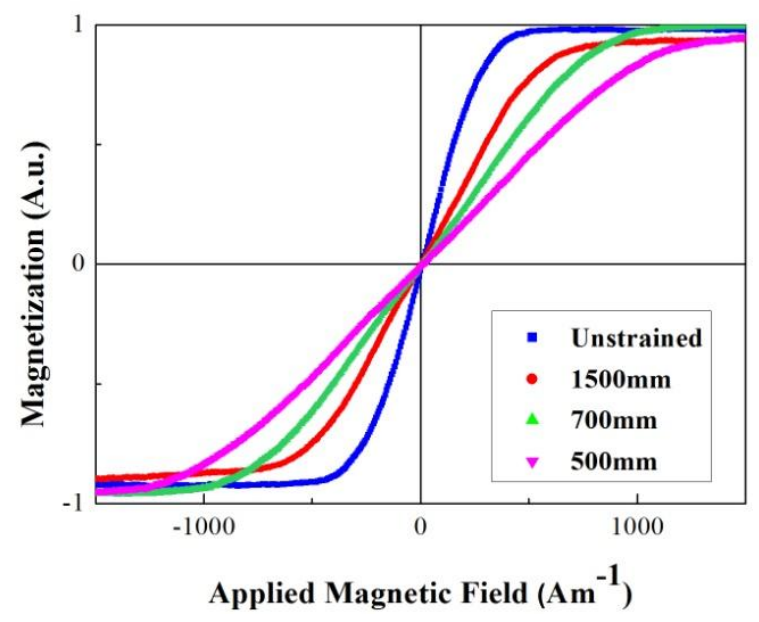

Figure 3:

Normalised magnetization of averaged hysteresis loops at different tensile stresses (or different radii of curvature) for $\mathrm{Ni}_{83} \mathrm{Fe}_{17}$ grown on $\mathrm{SiO}_{2}$ as a function of applied field.

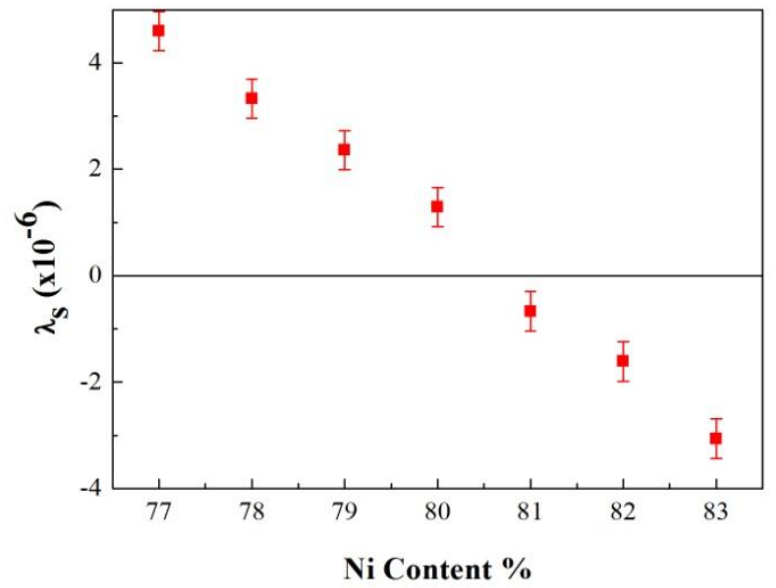

Figure 4:

Measured saturation magnetostriction $\lambda_{\mathrm{s}}$ as a function of Ni concentration (atomic \%). 


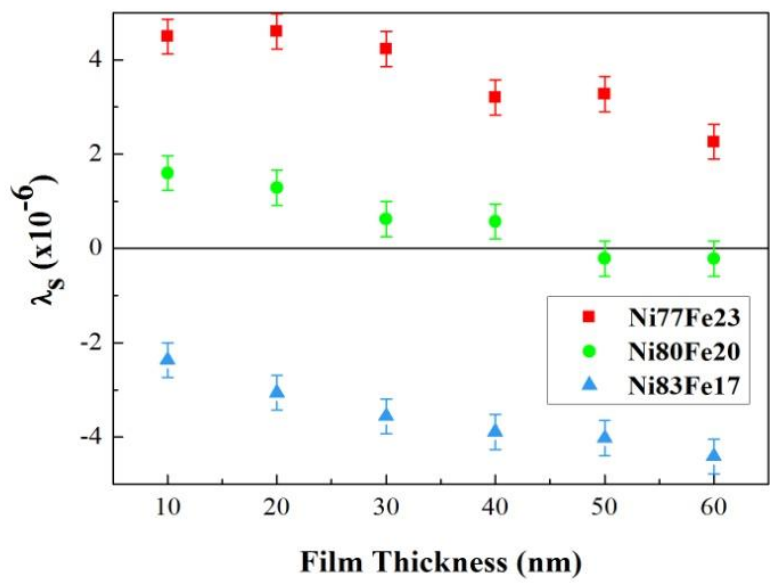

Figure 5:

Measured saturation magnetostriction $\lambda_{\mathrm{s}}$ as a function of film thickness for $\mathrm{Ni}_{77} \mathrm{Fe}_{23}, \mathrm{Ni}_{80} \mathrm{Fe}_{20}$ and $\mathrm{Ni}_{83} \mathrm{Fe}_{17}$.

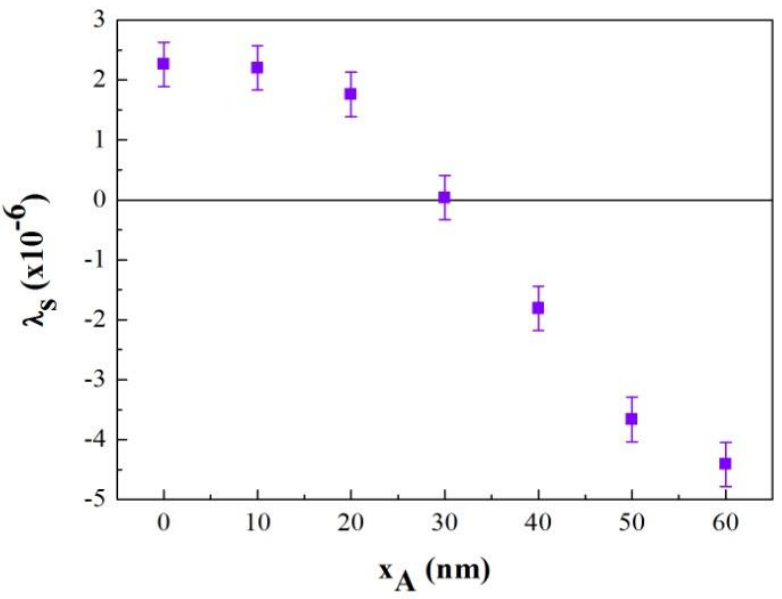

Figure 6:

Measured saturation magnetostriction $\lambda_{\mathrm{s}}$ of bi-layered samples of $\mathrm{A}(\mathrm{x} \mathrm{nm}) / \mathrm{B}(60-\mathrm{x} \mathrm{nm})$, where $\mathrm{A}$ is $\mathrm{Ni}_{83} \mathrm{Fe}_{17}$ and $\mathrm{B}$ is $\mathrm{Ni}_{77} \mathrm{Fe}_{23}$, as a function of $\mathrm{x}_{\mathrm{A}} \mathrm{nm}$. 
[1] Osaka T, Takai M, Hayashi K, Ohashi K, Saito M and Yamada K 1998 A soft magnetic CoNiFe film with high saturation magnetic flux density and low coercivity Nature $392796-798$

[2] Okita K, Ishiyama K and Miura H 2012 Magnetostriction measurement of a giant magnetoresistance film on a practical substrate covered by a shield layer J. Appl. Phys. 111 07E340-3 [3] Kudo K, Oikawa G, Maruyama Y, Saiki N and Shiina H 2008 Magnetic Shield, Manufacturing Method Thereof and Thin Film Magnetic Head Employing the Same U.S. Patent No. US2008/0002308 Al

[4] Hunter D et al 2011 Giant magnetostriction in annealed $\mathrm{Co}_{1-\mathrm{x}} \mathrm{Fe}_{\mathrm{x}}$ thin-films Nat. Commun. 2518 [5] Squire P T 1994 Magnetomechanical measurements of magnetically soft amorphous materials Meas. Sci. Technol. 5 67-81

[6] Raghunathan A, Snyder J E and Jiles D C 2009 Comparison of Alternative Techniques for Characterizing Magnetostriction and Inverse Magnetostriction in Magnetic Thin Films IEEE Trans. Magn. 45 3269-73

[7] Weber M, Koch R and Rieder K H 1994 UHV Cantilever Beam Technique for Quantitative Measurements of Magnetization, Magnetostriction, and Intrinsic Stress of Ultrathin Magnetic Films Phys. Rev. Lett. 73 1166-1169

[8] Wang B W, Li S Y, Zhou Y, Huang W M and Cao S Y 2008 Structure, magnetic properties and magnetostriction of $\mathrm{Fe}_{81} \mathrm{Ga}_{19}$ thin films J. Magn. Magn. Mater. 320 769-773

[9] Rengarajan S, Lee B H, Choe G and Walser R M 1995 Measurement of Very Low Magnetostrictions in Thin Films IEEE Trans. Magn. 31 3391-3393

[10] Claassen J H, Willard M A and Harris V G 2002 A simple system to measure magnetostriction in soft magnetic materials Rev. Sci. Instrum. 73 3265-7

[11] Harllee III P S, Bellesis G H and Lambeth D N 1994 Anisotropy and magnetostriction measurement by interferometry J. Appl. Phys. 75 6884-6

[12] Ahn S J, Kim D W, Kim H S, Kim C G, Lee Y S and Cho J 2001 Annealing Effect and Measurement of Magnetostriction in $\mathrm{Fe}_{49} \mathrm{Co}_{49} \mathrm{~V}_{2}$ Alloy using the Michelson Laser Interferometer Jpn. J. Appl. Phys. $403180-3182$

[13] Grossinger R Accurate Measurement of the Magnetostriction of Soft Magnetic Materials, Technical Report, Institute of Experimental Physik, Tech. Univ. Vienna, Austria

[14] Ekreem N B, Olabi A G, Prescott T, Rafferty A and Hashmi M S J 2007 An overview of magnetostriction, its use and methods to measure these properties J. Mater. Process. Technol. 191 96101

[15] Katada H, Shimatsu T, Watanabe I, Muraoka H, Nakamura Y and Sugita Y 2001 Induced uniaxial magnetic anisotropy and film magnetostriction in very thin permalloy films IEEE Trans. Magn. 37 2334-6 
[16] Kundys B, Bukhantsev Y, Vasiliev S, Kundys D, Berkowski M and Dyakonov V P 2004 Three terminal capacitance technique for magnetostriction and thermal expansion measurements Rev. Sci. Instrum. 75 2192-6

[17] Wetherhold R C and Chopra H D 2001 Beam Model for Calculating Magnetostriction Strains in Thin Films and Multilayers Appl. Phys. Lett. 79 3818-3820

[18] Rotter M, Muller H, Gratz E, Doerr M and Loewenhaupt M 1998 A miniature capacitance dilatometer for thermal expansion and magnetostriction Rev. Sci. Instrum. 69 2742-2746

[19] Schmiedeshoff G M et al 2006 Versatile and Compact Capacitive Dilatometer Rev. Sci. Instrum. 77 123907-1-7

[20] Choe G and Megdal B 1999 High precision magnetostriction measurement employing the B-H looper bending method IEEE Trans. Magn. 35 3959-61

[21] Hollingworth M P, Gibbs M R J and Murdoch S J 2003 Magnetostriction and surface roughness of ultrathin NiFe films deposited on $\mathrm{SiO}_{2} J$. Appl. Phys. 94 7235-9

[22] Chen K and Brennan F P 1998 A theoretical and experimental study of alternating current stress measurement under different loading modes J. Strain Anal. Eng. 33 291-303

[23] Datta S, Atulasimha J and Flatau A B 2007 Modelling of magnetostrictive Galfenol sensor and validation using four point bending test J. Appl. Phys. 101 09C521-3

[24] Kundys B, Bukhantsev Yu, Szymczak H, Gibbs M R J and Zuberek R 2002 Temperature dependence of saturation magnetostriction measured for $\mathrm{Fe}_{81} \mathrm{Si}_{3.5} \mathrm{~B}_{13.5} \mathrm{C}_{2}$ J. Appl. Phys. 351095 1098

[25] Gehring G A, Ali M, Cooke M D and Mattheis R 2003 Determination of magnetostriction in thin films using strained substrate techniques J. Magn. Magn. Mater. 257 15-21

[26] Jay J-P, Le Berre F, Pogossian S P and Indenbom M V 2010 Direct and inverse measurement of thin films magnetostriction J. Magn. Magn. Mater. 322 2203-14

[27] Sahingoz R, Hollingworth M P, Gibbs M R J and Murdoch S J 2005 Magnetoresistance and Magnetostriction of $\mathrm{Ni}_{81} \mathrm{Fe}_{19}$ and $\mathrm{Co}_{90} \mathrm{Fe}_{10}$ mono- and bilayer films J. Magn. Magn. Mater. 290-291 1059-1062

[28] Javed A, Szumiata T, Morley N A and Gibbs M R J 2010 An Investigation of the effect of Structural Order on Magnetostriction and Magnetic Behaviour of Fe-Ga alloy thin films Acta Materialia 58 4003-4011

[29] Song O, Ballentine C A and O'Handley R C 1994 Giant surface magnetostriction in polycrystalline Ni and NiFe films Appl. Phys. Lett. 64 2593-5

[30] Zhai H R, Xu Y B, Lu M, Miao Y Z, Hogue K L, Naik H M, Ahmad M and Dunifer G L 1991 Thickness and substrate dependence of the magneto-optical Kerr effect of thin magnetic films J. Appl. Phys. 70 5858-60

[31] Ali M and Watts R 1999 Measurement of saturation magnetostriction using novel strained substrate techniques and the control of the magnetic anisotropy J. Magn. Magn. Mater. 202 85-94 
[32] Hollingworth M P, Gibbs M R J and Hill E W 2003 Surface, interface and bulk studies of NiFe nanometer films for magnetoresistive heads J. Appl. Phys. 93 8737-9

[33] Penn T C and West F G 1966 Measuring Ferromagnetic Film Magnetostriction by Means of Magnetoresistance Rev. Sci. Instrum. 37 1137-9

[34] Jen S U, Wu T C, Lin C C and Chang K H 2004 Anisotropic magnetoresistance and magnetostriction of [Fe15Ni85/Fe25Ni75] and [Co35Ni65/Fe25Ni75] multilayers Solid State Communications 132 259-62

[35] Klokholm E and Aboaf J A 1981The saturation magnetostriction of permalloy films J. Appl. Phys. 52 2474-6

[36] Endo Y, Mitsuzuka Y, Shimada Y and Yamaguchi M 2011 Influence of magnetostriction on damping constant of $\mathrm{Ni}_{\mathrm{x}} \mathrm{Fe}_{1-\mathrm{x}}$ film with various $\mathrm{Ni}$ concentrations (x) J. Appl. Phys. 109 07D336-3 [37] Bonin R, Schneider M L, Silva T J and Nibarger J P 2005 Dependence of Magnetization Dynamics on Magnetostriction in NiFe alloys J. Appl. Phys. 98 123904-1-8

[38] Kawai T, Ouchi S, Ohtake M and Futamoto M 2012 Thickness Effect on Magnetostriction of Fe and $\mathrm{Fe}_{98} \mathrm{~B}_{2}$ Thin Films IEEE Trans. Magn. 48 1585-1558 